(D) Aline Muniz Cruz Tavares ${ }^{1}$

(D) Fernanda Ribeiro da Silva ${ }^{1}$

(D) Maria Auxiliadora Macedo Callou ${ }^{1}$

(D) Rejane Ferreira da Silva ${ }^{1}$

(D) Monise Pricila Salviano de Morais $^{1}$

(iD) Élida Mara Braga Rocha ${ }^{1}$

${ }^{1}$ Centro Universitário de Juazeiro do Norte - UNIJUAZEIRO, Núcleo de Estudos em Nutrição. Juazeiro do Norte, CE, Brasil

Correspondência Aline Muniz Cruz Tavares alinemunizcruz56@gmail.com

\section{Fatores que interferem na duração do aleitamento materno de crianças na Região Metropolitana do Cariri cearense}

\author{
Factors that interfere in the duration of breastfeeding of \\ children in the Metropolitan Region of Cariri, Ceará
}

\section{Resumo}

Introdução: O Aleitamento Materno Exclusivo (AME) é uma prática alimentar fundamental para a promoção da saúde materno-infantil. Porém, os índices para o AME no Brasil ainda se encontram abaixo do recomendado pela Organização Mundial da Saúde. Objetivo: Analisar os fatores que interferem na duração do aleitamento materno de crianças situadas na Região Metropolitana do Cariri cearense. Método: Estudo transversal descritivo, com abordagem quantitativa, realizado na Região Metropolitana do Cariri cearense. A amostra foi composta por 177 crianças entre seis e 23 meses de idade. Os dados foram coletados por meio de questionários, contendo informações a respeito das condições socioeconômicas e demográficas da família e da saúde infantil. As análises estatísticas foram realizadas com o software Statistical Package for the Social Sciences, procedendo-se a análise descritiva dos dados e aplicação do teste Qui-Quadrado. Razões de Chance (RC), intervalo de confiança de 95\% (IC95\%) e respectivos valores de p foram calculados pela regressão logística multinominal. Resultados: O tempo médio para o AME foi de 4,33 $( \pm 1,98)$ meses. Somente $38,9 \%$ das crianças avaliadas foram amamentadas, exclusivamente, até os seis meses de vida. Na análise de regressão logística multivariada verificou-se maior chance de tempo de AME adequado nas famílias com <1/4 salário mínimo (RC: 2,270 IC95\% 1,104 - 4,666, p= 0,026). Conclusão: O AME, nos municípios avaliados, ainda é insatisfatório de acordo com as recomendações preconizadas pelas entidades de saúde. A baixa renda per capita foi o fator determinante para uma adequada prática do AME na vida dos lactentes.

Palavras-chave: Aleitamento Materno. Desmame. Lactente. Estratégia de Saúde da Família.

\footnotetext{
Abstract

Introduction: Exclusive Breastfeeding (EBF) is a fundamental dietary practice for the promotion of maternal and child health. Unfortunately, the rates for EBF in Brazil are still below that recommended by the World Health Organization. Objective: To analyze the factors that interfere in the duration of breastfeeding of children living in the metropolitan region of Cariri, Ceará. Method: Descriptive cross-sectional study, with a quantitative approach, carried out in the Metropolitan Region of Cariri, Ceará. The sample consisted of 177 children between 6 and 23 months of age. The data were collected through questionnaires containing information about the socioeconomic and demographic conditions of the family and child health. Statistical analyses were
} 
performed using the software Statistical Package for the Social Sciences, proceeding to the data descriptive analysis, application of the Chi-Square test, Odds Ratios (OR), 95\% Confidence Interval (95\% Cl) and respective values of $\mathrm{p}$ were calculated by multinomial logistic regression. Results: The mean EBF length was $4.33( \pm 1.98)$ months, only $38.9 \%$ of the evaluated children were exclusively breastfed until 6 months of life. In the multivariate logistic regression analysis, a greater chance of adequate EBF length was verified in families with <1/4 minimum wage (OR: 2.270 95\% Cl $1.104-4.666, p=$ 0.026). Conclusion: EBF in the municipalities evaluated is still unsatisfactory according to the recommendations recommended by health entities. Low per capita income was the determining factor for the proper practice of EBF in the lives of infants.

Keywords: Breastfeeding. Weaning. Infant. Family Health Strategy. 


\section{INTRODUÇÃO}

O aleitamento materno exclusivo (AME) refere-se à prática de alimentar a criança somente com leite materno, não devendo ser ofertado nenhum outro alimento ou líquido, nem mesmo água, com exceção de medicamentos, soluções contendo vitaminas, sais de reidratação oral e suplementos minerais. A Organização Mundial da Saúde (OMS) recomenda que o AME seja realizado sob livre demanda por, no mínimo, seis meses de vida. ${ }^{1}$

Já está devidamente comprovada, pela literatura científica, que a amamentação proporciona benefícios para a saúde materno-infantil. O estudo de Andrade² demonstrou inúmeros benefícios do aleitamento materno tanto para o lactente quanto para a lactante. O aleitamento promove a interação mãe-filho, estimula o desenvolvimento facial infantil, que contribui para a mastigação, deglutição e respiração, além de promover proteção imunológica e ganho de peso adequado. Mães que amamentam apresentam risco menor de desenvolver câncer de mama e ovário, osteoporose e artrite reumatoide, além de proporcionar perda de peso mais rápida no período puerperal. ${ }^{3}$

Segundo o estudo de Boccolini et al., ${ }^{4}$ as prevalências para AME entre os menores de seis meses, AM e AME no primeiro ano de vida tiveram tendência ascendente, cujos principais ganhos foram observados entre 1986 e 2006. Já em 2013, houve redução da prevalência de AME entre os bebês de zero a dois meses de idade e os bebês de três a cinco meses de idade, em relação ao inquérito de 2006. Apesar do aumento significativo dos níveis dos indicadores de aleitamento materno ao longo do tempo, as recomendações quanto à duração do aleitamento materno e do AME ainda se encontram abaixo do recomendado pela OMS.

Apesar da existência de leis, programas e ações destinados à promoção, proteção e apoio ao Aleitamento Materno (AM), pesquisas recentes revelam níveis baixos para o AM no país, embora haja estudos que indicam que o AM previne o surgimento de doenças infecciosas e diarreicas, alergias e doenças crônicas não transmissíveis. ${ }^{1,5-7}$

A interrupção do AME acontece quando se introduzem ao lactente água, chás, sucos, leites não maternos ou outros alimentos líquidos, ou sólidos, antes deles completarem seis meses de vida. Essa prática errônea de oferecer alimentos precocemente configura-se como importante problema de saúde pública, podendo proporcionar aumento da morbimortalidade infantil, diminuição do consumo de leite materno e distúrbios nutricionais. 8,9

A literatura científica relata algumas barreiras que podem ocasionar a interrupção precoce do AME, dentre as quais se destacam o nível de escolaridade materna, uso de chupeta, tipo de parto, renda familiar, idade materna, retorno ao trabalho, baixo senso de coerência materna, interferências mamares, herança cultural, déficit de conhecimentos interligados à insegurança materna e percepção materna de leite fraco. Essas barreiras favorecem a introdução precoce de alimentos, contribuindo, assim, para o desmame precoce. ${ }^{10-13}$

Um estudo transversal realizado em 2007 nas Unidades Básicas de Saúde (UBS), da cidade do Rio de Janeiro com 1.507 lactantes demostrou que a idade materna, a experiência prévia em amamentação e a situação conjugal estão relacionadas à introdução de líquidos às crianças menores de seis meses. O estudo sugere ainda que o conceito de AME ainda não é bem compreendido entre as nutrizes, o que pode estar relacionado à dificuldade de manter o AME até os seis meses. ${ }^{9,14}$

Apesar da reconhecida importância da prática do AME até o sexto mês de idade, os índices, no Brasil, ainda se encontram abaixo do recomendado segundo a II Pesquisa de Prevalência de Aleitamento Materno nas capitais brasileiras e Distrito Federal, ${ }^{15}$ onde a mediana obtida para o AME foi de 60,84 dias e 182,52 dias 
para o aleitamento materno total. A OMS e Ministério da Saúde preconizam seis meses para AME e até 24 meses para aleitamento materno total. Além disso, a pesquisa também revelou que a capital do Ceará apresenta a menor prevalência para o AME em menores de seis meses, em comparação com as demais capitais da Região Nordeste.

Partindo do que foi observado na literatura científica e levando em consideração os inúmeros fatores relacionados à interrupção precoce do $\mathrm{AME}$, a presente pesquisa reúne vários aspectos científicos com o intuito de responder ao seguinte questionamento: que fatores podem influenciar na duração do AM em crianças assistidas na ESF (Estratégias de Saúde da Família) de alguns municípios do Cariri cearense?

Apesar de a Lei n 11.265/2006 ${ }^{16}$ vedar a divulgação, exposições especiais, cupons de desconto ou preço abaixo do custo, prêmios, brindes, vendas vinculadas a outros produtos e apresentações especiais das fórmulas infantis para lactentes, observa-se, no cenário social, uma busca crescente por fórmulas industriais que forneçam praticidade e agilidade para alimentar os lactentes. Segundo a concepção de algumas mães, o leite materno é fraco por não sustentar a saciedade da criança por muito tempo quando comparado às fórmulas infantis. Diante de tais evidências, a hipótese levantada por esta pesquisa supõe que variáveis maternas, juntamente com as socioeconômicas, possam influenciar na duração do AM em crianças assistidas na ESF.

Pesquisas com tal temática são importantes para a saúde materno-infantil, visto que detectam fatores que influenciam a duração do AM em crianças menores de dois anos de idade da região do semiárido nordestino. Os resultados obtidos neste estudo poderão auxiliar os profissionais de saúde a investir em intervenções direcionadas à promoção do AME nas comunidades e unidades de saúde. Diante desse contexto, o presente estudo teve como objetivo analisar os fatores que interferem na duração do aleitamento materno de crianças situadas na Região Metropolitana do Cariri cearense.

\section{MÉTODO}

Trata-se de pesquisa transversal descritiva, com abordagem quantitativa, realizada nos municípios da Região Metropolitana do Cariri cearense, que se destaca por seu turismo religioso, tesouros paleontológicos e centros universitários. O aeroporto Orlando Bezerra de Menezes está localizado estrategicamente para atender às regiões centro e sul do Ceará, sendo um dos principais instrumentos para o desenvolvimento econômico da região. Este surgiu a partir da conurbação entre os municípios de Juazeiro do Norte, Crato e Barbalha, chamado triângulo CRAJUBAR, sendo posteriormente incluídos os municípios limítrofes de Caririaçu, Farias Brito, Jardim, Missão Velha, Nova Olinda e Santana do Cariri.

O processo de amostragem probabilística considerou o sorteio de dois municípios da Região Metropolitana do Cariri cearense, sendo Barbalha a representação central e Missão Velha a periférica da região. O município de Barbalha possui 20 Estratégias de Saúde da Família (ESFs), estando 10 localizadas na zona rural e 10 na zona urbana, enquanto o município de Missão Velha possui 17 ESFs, sendo 11 situadas na zona rural e 06 na zona urbana.

A amostra do estudo foi composta por mães/responsáveis das crianças assistidas pela Atenção Básica em Saúde da Região Metropolitana do Cariri cearense. Somente as ESFs situadas na zona urbana foram incluídas na amostra, uma vez que as da zona rural têm difícil acesso. As informações referentes ao número de crianças acompanhadas e atendidas nas consultas de puericultura foram disponibilizadas pelas Secretarias de Saúde de ambos os municípios. 
Diante dessas informações, aplicaram-se um erro amostral de 5\% e um intervalo de confiança de 95\%, além do acréscimo de 10\% para perda amostral, o que totalizou uma amostra final de 177 crianças, sendo 88 da cidade de Missão Velha e 89 crianças da cidade de Barbalha. O processo de amostragem considerou a livre demanda, isto é, foram incluídas na pesquisa todas as mães/responsáveis convidadas que quiseram participar até alcançar a amostra.

Foram incluídas, na pesquisa, mães com idade igual ou superior a 18 anos, com crianças de seis meses a 24 meses de vida e que assinaram o Termo de Consentimento Livre e Esclarecido (TCLE). Foram excluídas do estudo as mães com crianças menores de seis meses de vida, mães adolescentes, com restrições físicas ou mentais que impossibilitassem a compreensão da investigação e capacidade para responder aos questionários em questão.

A fim de garantir o cumprimento das questões éticas e legais, o estudo foi submetido ao Comitê de Ética em Pesquisa da Faculdade de Juazeiro do Norte, e aprovado sob o parecer n 2.920 .644 . Os sujeitos da pesquisa foram previamente esclarecidos, em linguagem acessível, sobre os objetivos da investigação, a forma de coleta de dados, confidencialidade das informações, desconforto, liberdade de recusar durante o processo de coleta dos dados e publicação dos resultados da investigação, preservando a confidencialidade.

Após conhecer os objetivos, riscos e benefícios do estudo, os indivíduos que decidiram pela concordância na participação, assinaram o TCLE. Utilizou-se uma sala reservada na ESF para realização do estudo, a fim de evitar possível constrangimento na aplicação dos questionários, garantindo, assim, o sigilo e conforto dos colaboradores durante a pesquisa.

A coleta de dados ocorreu de setembro de 2018 a janeiro de 2019, sendo realizada mediante entrevista com acompanhantes das crianças cadastradas e acompanhadas pelas ESFs. Os dados foram coletados posteriormente às consultas de puericultura, por meio de um questionário pré-codificado e semiestruturado, contendo informações a respeito das condições socioeconômicas e demográficas da família e saúde infantil. As informações sobre consumo alimentar foram coletadas através de um instrumento formulado por Oliveira et al. ${ }^{17}$ para monitoramento da alimentação complementar entre crianças menores de dois anos. Uma equipe treinada e capacitada de estudantes de Nutrição aplicou os questionários, com duração média de 15 minutos, tendo como supervisão de trabalho uma pesquisadora com experiência em estudos epidemiológicos.

O questionário contendo informações socioeconômicas e demográficas incluía perguntas como: idade da mãe/responsável, estado civil, escolaridade em anos de estudos, participação em Programa Bolsa Família, renda familiar, número de integrantes maiores e menores de 18 anos de idade, acesso à luz elétrica, abastecimento da água, tratamento doméstico da água para beber, tratamento do esgoto e coleta de lixo. 0 questionário referente à saúde infantil incluía perguntas referentes à peso ao nascer, peso e estatura, tempo de aleitamento materno total e exclusivo.

O estudo adotou como variável dependente o indicador AME em menores de seis meses de vida (sim ou não), e como variáveis independentes do sexo da criança (feminino ou masculino), peso ao nascer ( $\geq 2.500$ ou >2.500 gramas), idade materna (20 a 34 anos ou $<20$ e >34 anos), escolaridade materna (<12 ou >=12 anos de estudo) e tabagismo durante gestação (sim ou não).

As análises estatísticas foram realizadas com o auxílio do software Statistical Package for the Social Sciences (SPSS) versão 20.0. Primeiramente, procedeu-se à análise descritiva dos dados, na qual foi realizada a distribuição de frequência das variáveis, sendo estes dados apresentados em tabelas. Em seguida, foi aplicado o teste Qui-Quadrado com o intuito de analisar a associação existente entre as variáveis selecionadas com o tempo de aleitamento materno exclusivo. 
Realizou-se, ainda, uma análise bivariada entre o tempo de aleitamento materno exclusivo com as variáveis independentes. Foram calculadas as Razões de Chances brutas (RCb), intervalos de confiança (IC95\%) e respectivos valores de $\mathrm{p}$. Todas as variáveis dos respectivos níveis que apresentaram valores de $p<0,20$ foram inseridas no modelo de regressão logística multivariada. Desenvolveu-se a construção de tabelas para a apresentação dos resultados obtidos na amostra.

\section{RESULTADOS}

Participaram do estudo 185 responsáveis por crianças menores de dois anos de vida. Em relação às variáveis maternas, foi observada maior frequência para idade de 18 a 24 anos (50,3\%), escolaridade de 12 anos ou mais de estudo (50,8\%) e união estável $(71,4 \%)$. Quanto às variáveis econômicas, 75,1\% eram beneficiárias pelo programa de transferência de renda, sendo que o valor médio do benefício recebido foi $\mathrm{R} \$ 203,51$. Em relação à renda, verificou-se que 70,3\% apresentavam renda per capita menor ou igual a um quarto do salário-mínimo, enquanto que renda familiar média foi de $R \$ 912,41$ reais. Referindo-se às variáveis infantis, 57,3\% pertenciam ao sexo feminino, com idade mínima de seis meses e máxima de 23 meses; a idade média foi de 13,43 ( \pm 4,94) meses; 64,9\% das crianças nasceram com peso adequado. 0 tempo médio para o aleitamento materno exclusivo foi de $4,33( \pm 1,98)$ meses, e de aleitamento materno total foi 11 meses, sendo que somente $38,9 \%$ das crianças avaliadas foram amamentadas exclusivamente até os seis meses de vida (tabela 1).

Tabela 1. Perfil sociodemográfico das mães e características das crianças acompanhadas pelas Estratégias de Saúde da Família, Cariri cearense, 2020.

\begin{tabular}{|c|c|c|}
\hline Variável & $n$ & $\%$ \\
\hline \multicolumn{3}{|l|}{ Idade materna } \\
\hline 20 a 34 anos & 153 & 82,7 \\
\hline$<20$ e $>34$ anos & 32 & 17,3 \\
\hline \multicolumn{3}{|l|}{ Escolaridade em anos } \\
\hline$<12$ anos de estudo & 91 & 49,2 \\
\hline$>=12$ anos de estudo & 94 & 50,8 \\
\hline \multicolumn{3}{|l|}{ Estado civil } \\
\hline União não estável & 53 & 28,6 \\
\hline União estável & 132 & 71,4 \\
\hline \multicolumn{3}{|l|}{ Renda familiar } \\
\hline$<=1 / 2 \mathrm{SM}$ & 26 & 14,1 \\
\hline 1 a $1 / 2 \mathrm{SM}$ & 108 & 58,4 \\
\hline$>1 \mathrm{SM}$ & 51 & 27,6 \\
\hline \multicolumn{3}{|l|}{ Programa Social Bolsa Família } \\
\hline Sim & 139 & 75,1 \\
\hline Não & 46 & 24,9 \\
\hline \multicolumn{3}{|l|}{ IMC materno $\left(\mathrm{kg} / \mathrm{m}^{2}\right)$} \\
\hline Baixo-peso & 7 & 3,8 \\
\hline Eutrofico & 99 & 53,5 \\
\hline Sobrepeso & 62 & 33,5 \\
\hline Obesidade & 17 & 9,2 \\
\hline \multicolumn{3}{|l|}{ Sexo } \\
\hline Feminino & 106 & 57,3 \\
\hline Masculino & 79 & 42,7 \\
\hline \multicolumn{3}{|l|}{ Peso ao nascer } \\
\hline$<=2500$ & 7 & 3,8 \\
\hline$>2500$ & 178 & 96,2 \\
\hline \multicolumn{3}{|c|}{ Tempo do aleitamento materno exclusivo } \\
\hline$<6$ meses & 105 & 56,8 \\
\hline 6 meses & 72 & 38,9 \\
\hline$>6$ meses & 8 & 4,3 \\
\hline
\end{tabular}

IMC- índice de massa corporal 
A análise bivariável mostrou que houve associação estatística significativa $(p<0,05)$ entre as variáveis: idade e escolaridade materna, renda familiar per capita, tratamento da água, destino do esgoto (tabelas 2 e 3).

Tabela 2. Análise bivariada da associação entre as variáveis independentes infantis e maternas e o aleitamento materno exclusivo. Cariri cearense, 2020

\begin{tabular}{|c|c|c|c|}
\hline Variável & Aleitamen & o exclusivo & $p^{*}$ \\
\hline \multirow{2}{*}{\multicolumn{4}{|c|}{ VARIÁVEIS INFANTIS }} \\
\hline & & & \\
\hline Sexo & & & 0,727 \\
\hline Feminino & $47(44,3 \%)$ & $59(55,7 \%)$ & \\
\hline Masculino & $33(41,8 \%)$ & $46(58,2 \%)$ & \\
\hline Peso ao nascer & & & 0,424 \\
\hline$<=2500$ & $2(28,6 \%)$ & $5(71,4 \%)$ & \\
\hline$>2500$ & $78(43,8 \%)$ & $100(56,2 \%)$ & \\
\hline \multicolumn{4}{|l|}{ VARIÁVEIS MATERNAS } \\
\hline Idade materna & & & 0,043 \\
\hline 20 a 34 anos & $61(39,9 \%)$ & $92(60,1 \%)$ & \\
\hline$<20$ e $>34$ anos & $19(59,4 \%)$ & $13(40,6 \%)$ & \\
\hline Escolaridade materna & & & 0,048 \\
\hline$<12$ anos de estudo & $46(50,5 \%)$ & $45(49,5 \%)$ & \\
\hline$>=12$ anos de estudo & $34(36,2 \%)$ & $60(63,8 \%)$ & \\
\hline Tabagismo durante gestação & & & 0,132 \\
\hline Sim & $21(53,8 \%)$ & $18(46,2 \%)$ & \\
\hline Não & $59(40,4 \%)$ & $87(59,6 \%)$ & \\
\hline
\end{tabular}

* Teste qui-quadrado de Pearson

Tabela 3. Análise bivariada da associação entre as variáveis independentes socioeconômicas e domiciliares e o aleitamento materno exclusivo. Cariri cearense, 2020.

\begin{tabular}{|c|c|c|c|}
\hline \multirow[t]{2}{*}{ Variável } & \multicolumn{2}{|c|}{ Aleitamento materno exclusivo } & \multirow[t]{2}{*}{$p^{*}$} \\
\hline & Sim & Não & \\
\hline VARIÁVEIS SOCIOECONÔMICAS & & & \\
\hline Renda per capita & & & 0,028 \\
\hline$<=1 / 4 \mathrm{SM}$ & $63(48,5 \%)$ & $67(51,5 \%)$ & \\
\hline$>1 / 4 \mathrm{SM}$ & $17(30,9 \%)$ & $38(69,1 \%)$ & \\
\hline Programa Bolsa Família & & & 0,516 \\
\hline Sim & $62(44,6 \%)$ & $77(55,4 \%)$ & \\
\hline Não & $18(39,1 \%)$ & $28(60,9 \%)$ & \\
\hline Nº de pessoa por domicílio & & & 0,797 \\
\hline < 5 Indivíduos & $51(44,0 \%)$ & $65(56,0 \%)$ & \\
\hline$>=5$ Indivíduos & $29(42,0 \%)$ & $40(58,0 \%)$ & \\
\hline $\mathrm{N}^{0}$ de pessoas menor de 18 anos & & & 0,518 \\
\hline$<3$ Crianças & $56(41,8 \%)$ & $78(58,2 \%)$ & \\
\hline$>=3$ Crianças & $24(47,1 \%)$ & $27(52,9 \%)$ & \\
\hline Estado civil & & & 0,312 \\
\hline União não estável & $26(49,1 \%)$ & $27(50,9 \%)$ & \\
\hline União estável & $54(40,9 \%)$ & $78(59,1 \%)$ & \\
\hline
\end{tabular}


Tabela 3. Análise bivariada da associação entre as variáveis independentes socioeconômicas e domiciliares e o aleitamento materno exclusivo. Cariri cearense, 2020. (Cont.)

\begin{tabular}{|c|c|c|c|}
\hline \multirow{2}{*}{$\begin{array}{ll}\text { Variável } & \\
& \text { VARIÁVEIS DOMICILIARES }\end{array}$} & \multicolumn{2}{|c|}{ Aleitamento materno exclusivo } & \multirow[t]{2}{*}{$p^{*}$} \\
\hline & & & \\
\hline Luz elétrica & & & 0,846 \\
\hline Sim & $79(43,2 \%)$ & $104(56,8 \%)$ & \\
\hline Não & $1(50,0 \%)$ & $1(50,0 \%)$ & \\
\hline Acesso à água & & & 0,783 \\
\hline Rede pública & $78(43,1 \%)$ & $103(56,9 \%)$ & \\
\hline Poço/cacimba/cisterna & $2(50,0 \%)$ & $2(50,0 \%)$ & \\
\hline Tratamento na água & & & 0,030 \\
\hline Sim & $36(53,7 \%)$ & $31(46,3 \%)$ & \\
\hline Não & $44(37,3 \%)$ & $74(62,7 \%)$ & \\
\hline Lixo & & & 0,052 \\
\hline Coleta municipal & $63(47,7 \%)$ & $69(52,3 \%)$ & \\
\hline Incinerado/ céu aberto & $17(32,1 \%)$ & $36(67,9 \%)$ & \\
\hline Esgoto & & & 0,033 \\
\hline Rede pública & $62(48,4 \%)$ & $66(51,6 \%)$ & \\
\hline $\begin{array}{l}\text { Fossa séptica/ fossa rudimentar/ céu } \\
\text { aberto }\end{array}$ & $18(31,6 \%)$ & $39(68,4 \%)$ & \\
\hline
\end{tabular}

* Teste qui-quadrado de Pearson

Após a regressão, somente famílias com <1/4 salário mínimo (RC: 2,270,IC95\% 1,104 - 4,666, p= 0,026) permaneceram significativamente associadas ao aleitamento materno exclusivo adequado, segundo a tabela 4.

Tabela 4. Regressão logística multivariada e medidas de risco (razão de chances - RC) e intervalo de confiança (IC95\%) para os fatores associados à duração do aleitamento materno exclusivo adequado em crianças entre seis e 24 meses. Cariri cearense, 2020.

\begin{tabular}{lccc}
\hline Fatores de risco & RC bruta & RC ajustada & Valor de $p$ \\
\hline Idade materna & IC95\% & IC95\% & \\
Escolaridade materna & $2,204(1,014-4,790)$ & $1,803(0,767-4,235)$ & 0,176 \\
Renda per capita & $1,804(1,002-3,248)$ & $1,519(0,790-2,920)$ & 0,210 \\
Tratamento da água & $2,102(1,078-4,097)$ & $2,270(1,104-4,666)$ & 0,026 \\
Esgoto & $1,953(1,063-3,588)$ & $1,877(0,957-3,684)$ & 0,067 \\
Lixo & $2,035(1,055-3,927)$ & $1,670(0,728-3,830)$ & 0,226 \\
Tabagismo & $1,934(0,989-3,780)$ & $11,584(0,671-3,741)$ & 0,294 \\
\hline
\end{tabular}

$\mathrm{RC}=$ razão de chance; IC = intervalo de confiança.

\section{DISCUSSÃO}

O estudo indica que o AME foi praticado por aproximadamente duas a cada cinco mulheres cadastradas e acompanhadas pelas ESF dos municípios avaliados. Sabe-se que a baixa adesão ao aleitamento materno exclusivo favorece a introdução precoce de outros tipos de leite e demais fontes alimentares, o que pode ocasionar engasgos, má-oclusão dentária, atopia, excesso de peso, anemia, microhemorragias intestinais e comprometimento do crescimento e desenvolvimento da criança na primeira infância. ${ }^{1,18-20}$ 
Os dados encontrados para tempo de AME e aleitamento materno total mostram que os resultados se encontram muito abaixo do que é recomendado. A média do AME foi de 4,33 meses, enquanto para o aleitamento materno total foi 11 meses, ou seja, valores bem abaixo da preconização da OMS e Ministério da Saúde, que é de seis meses para AME e até 24 meses ou mais para aleitamento materno total. ${ }^{1}$

Em relação à idade materna, o estudo demonstrou que não houve associação significativa entre esta variável e o tempo de AME. Estudos anteriores informam que o desmame precoce é mais frequente em mulheres mais jovens quando comparadas às mulheres com idade avançada. Subtende-se, então, que mulheres mais velhas possuem maior experiência e estabilidade emocional em relação à vários aspectos que possam influenciar positivamente no aleitamento materno. ${ }^{21,22}$

Quanto à escolaridade materna e ao tempo de amamentação, observa-se que mães com maior escolaridade tendem a interromper o AME precocemente, favorecendo a introdução, prematuramente, de outros alimentos à dieta da criança. Essa associação inversamente proporcional pode ocorrer porque mães com maior nível educacional apresentam maiores chances de serem inseridas no mercado de trabalho, o que pode limitar o tempo de aleitamento materno exclusivo.

Alguns estudos mostraram resultados contrários ao encontrado nesta pesquisa, ou seja, o maior nível educacional materno foi considerado um fator protetor para o AME, já que mães com o nível educacional mais elevado possuem mais oportunidades de acesso às informações sobre as vantagens e benefícios do AME para a saúde do lactente e da lactante quando comparadas às mães com menor nível educacional.23-27

Ao analisar a relação entre o destino final do lixo e o AME, observa-se que não houve associação significativa entre estas variáveis, embora os achados do estudo não tenham evidenciado associação entre esses fatores. Os estudos de Escobar et al. ${ }^{23}$ e de Oliveira et al. ${ }^{28}$ revelam que famílias com melhores condições de abastecimento de água, coleta de lixo e esgotamento sanitário amamentam suas crianças por mais tempo.

O lixo doméstico gera grande preocupação, já que, na maioria das vezes, são descartados de maneira incorreta no meio social. No presente estudo, é possível observar condutas inadequadas em relação ao destino final do lixo, como incineração, enterramento ou despejo em céu aberto. Tais informações implicam deficiência na execução da coleta municipal, visto que infelizmente, há municípios que não têm sistemas adequados de coleta de lixo. O lixo descartado incorretamente causa mau cheiro e contribui para a proliferação de insetos e roedores, causando danos à saúde humana e à natureza, ${ }^{29,30}$ podendo ocasionar doenças transmissíveis de grande risco para a saúde infantil, como diarreias.

Em relação aos dados socioeconômicos, a renda per capita foi a única variável que apresentou um dos resultados mais significativos na regressão logística multinomial, sendo implícita a relação entre esta variável e o tempo de AME. Sendo assim, famílias que possuem renda per capita baixa têm duas vezes mais chances de permanecer e alcançar o tempo de AME adequado, segundo a análise estatística do estudo. A revisão bibliográfica de Faleiros et al. ${ }^{31}$ revela que mulheres de classe baixa amamentavam mais do que as de classe alta, em virtude das dificuldades econômicas encontradas para aquisição de outros gêneros alimentícios, principalmente a partir do sexto mês de vida. Resultados semelhantes foram encontrados no estudo de Wenzel \& Souza, ${ }^{32}$ que buscou identificar os fatores que determinam o aleitamento materno nas diferentes regiões brasileiras e concluiu que em todas as regiões avaliadas a maior renda também foi associada ao menor tempo de AM.

Acredita-se que famílias em situação de vulnerabilidade econômica consigam manter o AME adequado em virtude de o AM ser uma prática econômica e sustentável de alimentar uma criança pequena, além de 
ser uma forma de proteger seus filhos de uma alimentação inadequada pela restrição de alimentos ou falta de disponibilidade de adequação alimentar.

\section{CONCLUSÃO}

A média para o AME encontrada no estudo foi superior à média nacional para a Região Nordeste, embora ainda insatisfatória, segundo a recomendação preconizada pelos órgãos nacionais e internacionais. Em relação aos fatores associados ao tempo de AME, observa-se que mães com baixa renda per capita apresentaram maiores chances de alcançar duração do aleitamento materno exclusivo, conforme as recomendações da OMS.

Ações voltadas à promoção, adesão, manutenção, apoio e incentivo ao AME devem ser ampliadas e intensificadas em todos os extratos de renda da população, a fim de fortalecer práticas de AME. Por fim, espera-se que os achados desta pesquisa possam servir de aporte científico para futuras pesquisas em relação aos fatores que levam à interrupção precoce do AME.

\section{AGRADECIMENTOS}

À bolsa de iniciação científica concedida pelo Centro Universitário de Juazeiro do Norte - UNIJUAZEIRO, por meio do Programa de Bolsa de Iniciação Científica (PBIC-FJN).

\section{REFERÊNCIAS}

1. Brasil. Ministério da Saúde (MS). Secretaria de Atenção à Saúde. Departamento de Atenção Básica. Saúde da Criança: Nutrição infantil: aleitamento materno e alimentação complementar. Brasília: MS; 2009. (Série A. Normas e Manuais Técnicos, Cadernos de Atenção Básica, nº 23).

2. Andrade ISN. Aleitamento materno e seus benefícios: primeiro passo para a promoção saúde. Rev Bras Promoç Saúde. 2014; 27(2):149-50

3. Caminha MFC, Serva VB, Arruda IKG, Batista Filho M. Aspectos históricos, científicos, socioeconômicos e institucionais do aleitamento materno. Rev Bras Saude Mater Infant 2010; 10(1):25-37.

4. Boccolini CS, Boccolini, PMM, Monteiro FR, Venâncio SI, Giugliani ERJ. Tendência de indicadores do aleitamento materno no Brasil em três décadas. Rev. Saúde Pública. 2017; 51: 108. http://dx.doi.org/10.11606/s1518-8787.2017051000029.

5. Takahashi, K.; Ganchimeg, T.; Ota, E.; Vogel, J.P.; Souza, J.P.; Laopaiboon, M.; Castro, C.P.; Jayaratne, K.; Ortiz-Panozo, E.; Lumbiganon, P.; et al. Prevalence of early initiation of breastfeeding and determinants of delayed initiation of breastfeeding: Secondary analysis of the WHO Global Survey. Sci. Rep. 2017; 7: 44868. DOI:10.1038/srep44868.

6. Gupta PM, Perrine CG, Chen J, Elam-Evans LD, Flores-Ayala R. Monitoring the World Health Organization Global Target 2025 for Exclusive Breastfeeding: Experience from the United States. J Hum Lact 2017; 33(3):578-581. DOI:10.1177/0890334417693210.

7. Garcia Ana Rita. Influência do Aleitamento Materno na Prevenção da Obesidade em Idade Pediátrica. Nutrícias [Internet]. 2013 Mar; (16): 26-29. Disponível em: http://www.scielo.mec.pt/scielo.php?script=sci_arttext\&pid=S2182-72302013000100005\&lng=pt.

8. Germoglio RG. Avaliação Da Introdução Precoce Da Alimentação Complementar Em Crianças Menores De 6 Meses Em João PessoaPB. Dissertação (Monografia - Graduação) Universidade Federal da Paraíba, 2014.

9. Parizoto GM, Parada CMGL, Venâncio SI, Carvalhaes MABL. Tendência e determinantes do aleitamento materno exclusivo em crianças menores de 6 meses. J Pediatr. 2009; 85(3):201-8 http://dx.doi.org/10.1590/S0021-75572009000300004. 
10. Cortelo FM, Marba ST, Cortellazzi KL, Ambrosano GM, Guerra LM, Almeida AC, et al. Women's sense of coherence and its association with early weaning. J Pediatr (Rio J). 2018; 94:624-9. http://dx.doi.org/10.1016/j.jped.2017.08.007.

11. Oliveira CS, locca FA, Carrijo MLR, Garcia RATM. Amamentação e as intercorrências que contribuem para o desmame precoce. Rev Gaúcha Enferm. 2015; 36(esp):16-23. http://dx.doi.org/10.1590/1983-1447.2015.esp.56766.

12. Bastian DP, Terrazzan AC. Tempo de aleitamento materno e os fatores de risco para o desmame precoce. Nutrire. 2015 Dec; 40(3):278-286. http://dx.doi.org/10.4322/2316-7874.49914

13. Santos PV, Martins MCC, Tapety Fl, Paiva AA, Fonseca FMNS, Brito AKS. Desmame precoce em crianças atendidas na Estratégia Saúde da Família. Rev. Eletr. Enf. [Internet]. 2018 [acesso em: abr 2018]; 20:v20a05. http://doi.org/10.5216/ree.v20.43690.

14. Niquini RP, Bittencourt SA, Lacerda EM, Oliveira MI, Leal MC. Acolhimento e características maternas associados à oferta de líquidos a lactentes. Rev Saude Publica. 2010; 44:677-85.

15. Brasil. Ministério da Saúde (MS). II Pesquisa de prevalência de aleitamento materno nas capitais brasileiras e Distrito Federal. Brasília: MS; 2009.

16. Brasil. Lei n 11.265, de 3 de janeiro de 2006. O Congresso Nacional Regulamenta a Comercialização de Alimentos para Lactentes e Crianças de Primeira Infância e também a de Produtos de Puericultura Correlatos. Diário Oficial da União. 04 jan. 2006 ; Seção 1

17. Oliveira JM, Castro IRRD, Silva GB, Venancio SI, Saldiva, SRDM. Assessing complementary feeding practices in the first two years of life: a proposal for indicators and a monitoring tool. Cad. Saúde Pública 2015; 31(2): 377-394. Doi: 10.1590/0102-311X00209513

18. Giugliani, ERJ. Amamentação exclusiva. In: Amamentação: bases científicas. 3a ed. Rio de Janeiro: Guanabara Koogan; 2010.

19. Siega-Riz AM, Deming DM, Reidy KC, Fox MK, Condon E, Briefel RR. Food consumption patterns of infants and toddlers: where are we now? J Am Diet Assoc 2010; 110:S38-51. DOI: 10.1016/j.jada.2010.09.001.

20. Balaban G, Silva GAP. Efeito protetor do leite materno contra a obesidade infantil.J Pediatr (Rio J). 2004; 80(1):7-16. DOI: 10.1590/S0021-75572004000100004.

21. Santana GS, Giugliani ER, Vieira TO, Vieira GO. Factors associated with breastfeeding maintenance for 12 months or more: a systematic review. J Pediatr (Rio J). 2018; 94(2):104-22. http://dx.doi.org/10.1016/j.jped.2017.06.013.

22. Villar M, Santa-Marina L, Murcia M, Amiano P, Gimeno S, Ballester F, et al. Social Factors Associated with Non-initiation and Cessation of Predominant Breastfeeding in a Mother-Child Cohort in Spain. Matern Child Health J. 2018; 22(5):725-34. DOI: 10.1007/s10995018-2441-1

23. Escobar AMU, Ogawa AR, Hiratsoka M, Kawashita MY, Teruya PY, Grisi S, et al. Aleitamento materno e condições sócio-econômicoculturais: fatores que levam ao desmame precoce. Rev Bras Saúde Mater Infant. 2002; 2(2):253-61. http://dx.doi.org/10.1590/S1519-38292002000300006.

24. Damião Jj. Influência da escolaridade e do trabalho maternos no aleitamento materno exclusivo. Rev Bras Epidemiol. 2008; 11(3):442-52. DOI:10.1590/S1415-90X200800030001

25. Kornides M, Kitsantas P. Evaluation of breastfeeding promotion, support, and knowledge of benefits on breastfeeding outcomes. J Child Health Care. 2013 Sep; 17(3):264-73. http://dx.doi.org/10.1177/1367493512461460.

26. Wijndaele K, Lakshman R, Landsbaugh JR, Ong KK, Ogilvie D: Determinants of early weaning and use of unmodified cow's milk in infants: a systematic review. J Am Diet Assoc. 2009; 109(12):2017-2028. DOI: 10.1016/j.jada.2009.09.003.

27. Boff ADG, Paniagua LM, Scherer S, Goulart BNG. Aspectos socioeconômicos e conhecimento de puérperas sobre aleitamento materno. Audiol Commum Res. 2015; 20(2):141-145. http://dx.doi.org/10.1590/S2317-64312015000200001517.

28. Oliveira MGOA, Lira PIC, Batista Filho M, Lima MC. Factors associated with breastfeeding in two municipalities with low human development index in Northeast Brazil. Rev Bras Epidemiol. 2013; 16(1):178-89. http://dx.doi.org/10.1590/S1415$790 \times 2013000100017$ 
29. Silva, ARS; Melo, DG; Morales, FJS; Antônio, T; Coelho, TPM; Silva, GS. Impactos ambientais referentes à não coleta de lixo e reciclagem. Ciências Exatas e Tecnológicas. 2015; 2(3):63-76.

30. Vianna, AM. Poluição ambiental, um problema de urbanização e crescimento desordenado das cidades. Revista Sustinere. 2015; 3(1):22-42. doi:https://doi.org/10.12957/sustinere.2015.17325.

31. Faleiros FTV, Trezza EMC, Carandina L. Aleitamento materno: fatores de influência na sua decisão e duração. Rev Nutr 2006; 19(5):623-30. https://doi.org/10.1590/S1415-52732006000500010

32. Wenzel D, Souza SB. Fatores associados ao aleita-mento materno nas diferentes Regiões do Brasil. Rev Bras Saúde Matern Infant. 2014; 14(3):241-9. http://dx.doi.org/10.1590/S1519-38292014000300005

\section{Colaboradoras}

Tavares AMC participou da coleta de dados, análise estatística, escrita e revisão final do manuscrito; Silva FR participou da coleta de dados, análise estatística e revisão final; Silva RF participou da coleta de dados e revisão final; Callou MAM participou do planejamento do projeto de pesquisa e revisão final do manuscrito; Morais MPS participou da coleta de dados e análise estatística; Rocha EMB participou do planejamento do projeto de pesquisa, análise estatística, escrita e revisão crítica do manuscrito para submissão.

Conflito de Interesses: Os autores declaram não haver conflito de interesses.

Recebido: 16 de dezembro de 2019

Aceito: 06 de julho de 2020 